\title{
Teaching Dynamics Using Sports Biomechanics
}

\author{
Sean Maw \\ Mount Royal University, Calgary, Alberta, Canada \\ Corresponding Author E-mail Address: smaw@mtroyal.ca
}

\begin{abstract}
The first course in Dynamics can be a challenging one for many undergraduate engineering students. Concepts can be complex, mathematical treatments can be non-trivial, and the theory can be difficult to apply. While lectures introduce course material, tutorials are often used for textbook problem solving and labs allow for an experiential exploration of the concepts. However, given the volume of material covered in Dynamics, the relative infrequency of labs, and their limited duration, one could argue that labs do not adequately fulfill their important role. With this perspective in mind, a redesign of this course took place so that in each lecture, one or more concepts were visually demonstrated and/or students took part in an activity to illustrate the concepts. This was in addition to the regular lab experiences. Furthermore, many of the demonstrations were taken from the world of sports to provide both accessible relevance and reinforcing examples. Feedback from students suggests that this approach is both helpful and motivating. Students report "getting it" through these demonstrations and enjoying the learning experience more than conventional approaches. Students were asked which "daily dynamic demos" they preferred, and the reasons for those preferences as a matter of course development. It appears that the richer, more personally relevant and more interactive the demonstrations, the more impact they had. This paper describes the various demonstrations and lab exercises, the motivations behind using each of them, and the concepts that were illustrated by each one. Preferred ones are identified, and the reasons for those preferences are also presented.
\end{abstract}

Keywords: dynamics, demonstrations, sports, pedagogy, biomechanics, labs

\section{INTRODUCTION}

Dynamics is a challenging course for many $1^{\text {st }}$ or $2^{\text {nd }}$ year engineering students. Covering particle and rigid body kinematics and kinetics, the course spans a broad range of theoretical concepts and an even greater range of problems for students to solve. For some students, instruction based solely on theory and problem solving can result in low motivation levels and frustration over relevance.

In an effort to make the course more relevant to his students, the author introduced three new elements to the course; a topical theme, daily dynamic demonstrations of concepts, and (usually) practical lab exercises. The stated theme of the course was sports. For almost all lectures during the term, some form of demonstration of a concept took place. Some demonstrations were simply presented while others were interactive. The labs were intended to allow for a more free-form exploration of concepts, where problems were not as clearly stated as they would be in the textbook.

The reason these changes were introduced to the course was to increase motivation levels, to help students apply the learned Dynamics concepts to their own life experiences, and to develop an appreciation for how Dynamics affects so many aspects of their lives.

This paper describes the demonstrations, lab exercises and theme application to a level of detail sufficient to allow the reader to adopt them. The author offers reflections on the perceived value of the course components and draws upon feedback from the students in the form of an end-of-term survey. The survey was conducted in a manner consistent with ethical research practices. However, the survey was undertaken simply as a quality improvement activity for teaching and did not involve any formal research methodology. Nevertheless, as a description of teaching activities and as a reflection upon said activities, the author hopes that the reader may find value in a review of the story.

\section{COURSE CHARACTERIZATION}

\subsection{Logistics}

The Dynamics course was taught in the Winter term (Jan-Apr, 2013) at Mount Royal University (MRU), Calgary, Alberta. The MRU program is a transfer program where most students transfer to the University of Calgary, Alberta or Saskatchewan. About a third of the 
class was hoping to attend the UofA, and was taking a heavy load of other courses. Most of the other students were in their last term of a 1.5 to 2 year stay at MRU, and were carrying 3-4 courses. Twenty-two students completed the course.

Each of 13 weeks, there were two 80 minute lecture periods held mid-day on Tuesdays and Thursdays, one 80 minute tutorial period held early Friday morning, and every other week there was a 120 minute Thursday evening lab period. Typically, lectures consisted of a discussion of the relevant theory from the text, a daily dynamic demo, and then some problem solving from the relevant portion of the text. The first half of the term was more theory-laden while the latter half had a heavier emphasis on problem solving. The tutorials typically focused on problem solving, although quizzes were held in the tutorial periods.

The text was Meriam \& Kraige's $6^{\text {th }}$ SI edition [1]. In terms of course evaluation, six assignments were worth $15 \%$, three quizzes were worth $10 \%$, six labs were worth $15 \%$, a project was worth $10 \%$, a midterm was worth $15 \%$ and the final exam was worth $35 \%$ (which had to be passed to get more than a $\mathrm{D}+$ in the course).

\subsection{Objectives}

The course content emphasizes kinematics and kinetics of particles and rigid bodies, rectilinear and curvilinear motion, general planar motion, rotation, relative motion, force, mass, acceleration, friction, impulse, momentum and conservation of linear and angular momentum. By the end of the course, students should be able to analyze particles and rigid bodies in two dimensions, using concepts of dynamic equilibrium, impulse, impact and momentum, and work and energy under the action of static or dynamic friction, as required. They should also be able to calculate moments of inertia (MOI) and use them in solving rigid body problems. Use of proper terminology and notation was evaluated throughout the course. Students were also expected to be able to describe real-life situations and problems in writing, and verbally, using correct terms and reasoning.

\subsection{The Sports Theme}

From the outset, it was made clear that "sports" would be a prominent theme in the course. The purpose of adopting this theme was to engage the students in something that they related to and liked. A wide range of sport and recreation examples were touched upon, including some from running, skating, swimming, hockey, discus, pool, basketball, volleyball, curling, skiing, baseball, weights, soccer, golf, and tennis. That said, the sport references were more frequent in the first half of the term, which was noted by some students (who wished the theme had been maintained more consistently to the end).

Aside from sport references during lectures and demonstrations, some (though not many/enough) were incorporated into labs. Most quizzes, as well as the midterm and final, referred to one or more sport situations. Many questions on assignments had a sport/recreation connection, at least for the first $2 / 3$ rds of the term.

\subsection{Lectures and Daily Dynamic Demos}

Each lecture began with administrative announcements, followed either by a daily dynamic demonstration (DDD) of a key concept or by lecture coverage of a specific theory topic. If the lecture followed right after the announcements, then a demonstration was nested within the lecture component.

The following list is a brief description of the 15 most popular demonstrations:

- Ball drop and bounce - a tennis ball was dropped from a height and it bounced off the floor - the first DDD of the term, students were asked to explain everything that was going on as best they could

- Rolling balls, bouncing off walls - a ball was kicked (against a wall in the $2^{\text {nd }}$ kick) and students were asked to sketch position, velocity and acceleration versus time (plus acceleration versus position) - they enjoyed the challenge of this one

- Ball throwing - a ball was tossed to someone at various launch angles - students decided on what the key parameters were and their independence

- Taking a basketball pass - a basketball was passed to the instructor moving at various speeds, to illustrate practical relative motion considerations

- Instructor on a pulley - a rope was arranged in a horizontal pulley arrangement with the instructor being the load - a student pulled on the rope, illustrating key characteristics of the governing rope equations

- Throwing a ball off the wall - similar to the first DDD, students were asked to describe all relevant forces/concepts (details of impacts being new)

- Mogul skiing - students watched an instructional YouTube video on skiing moguls and evaluated the advice from a dynamics perspective - this was very popular as most students had done some skiing

- Leg and arm curls - students watched another pair of videos on weight training, and were asked to explain what was happening, illustrating the value of using work and energy methods

- Soccer ball kick - illustrating the merit and value of key concepts in impact, impulse and momentum methods, a ball was kicked and students were asked how to best analyze the situation 
- Curling - another instructional YouTube video was shown, to illustrate the differences in considering a mass as a particle versus a rigid body

- Lunch box of golf balls - a transparent lunch box containing golf balls was raised, lowered and shaken, to illustrate the differences in internal and external forces for a system of particles, as well as mass center and constituent particle dynamics

- Rolling LED wheel - a bicycle wheel with an LED on the rim was rolled down the hall - this nicely demonstrated the instantaneous center (IC) of zero velocity as well as the motion of a point on a wheel through space - students sketched what they saw

o Rolling chalk wheel on blackboard - a disk with bits of chalk stuck on it was rotated back and forth while pressed against a blackboard - the lines normal to these short arcs converge at the IC, to illustrate how the IC can be located
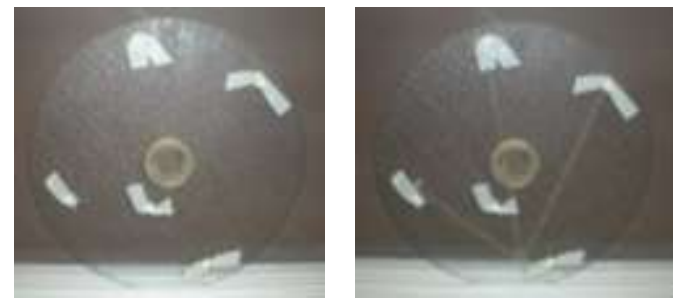

Figure 1 - Rolling chalk wheel: perpendiculars all converge on the instantaneous center

- Trick spindle - a spindle with a rope wound about it was pulled - students were asked to guess which direction it would move - the answer depends on the angle of the pull as determined by the moment around the IC
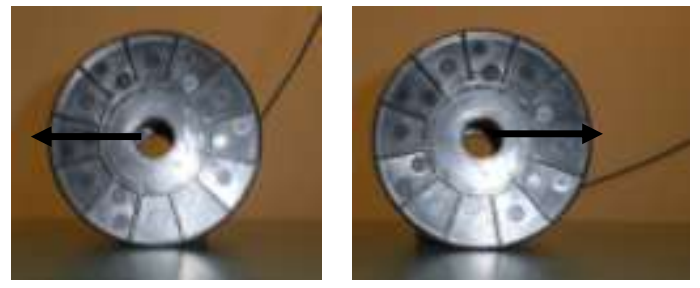

Figure 2 - Trick spindle: pull rope up, spindle moves left; pull rope to side, spindle moves right

- Spinning Meccano ${ }^{\mathrm{TM}}$ about an axis with a given force - by changing the MOI of the Meccano ${ }^{\mathrm{TM}}$ piece that is spinning, the idea of angular momentum was constructed

- Spinning Meccano ${ }^{\mathrm{TM}}$ in the air - a rigid set of links were tossed into the air, twirling and then not twirling, to appreciate the difference between the motion of the CoG and the motion of the body's components around the $\mathrm{CoG}$

\subsection{Labs}

Approximately every two weeks, a 2 hour lab was held where students would be given various tasks to complete. Generally, they completed them within the lab period, and then they would go home to write them up over the subsequent week. Lab reports typically consisted of an explanation of the lab's purpose, a description of materials and methods, a theoretical derivation, and a discussion of successes, assumptions, challenges faced, and lessons learned. Most labs involved 2-3 different activities, and they were completed in groups of 4-5 students.

This following list provides a brief description of the 10 most prominent lab exercises:

- Experiencing 1 Newton - students had to create a situation where they were applying $\approx 1 \mathrm{~N}$ to bring about the acceleration of a mass, and then they had to prove that that was what they had done

- Rocket launch - students had to launch projectiles in rectilinear and curvilinear fashion and analyze various details about the trajectory using limited measuring equipment

- Angry Birds ${ }^{\mathrm{TM}}$ - students analyzed the video game for the use of basic dynamics concepts (projectile flight, KE, momentum, friction, conservation)

- Track running - students analyzed 3D forces and accelerations involved in running the corners around the campus track

○ Climbing stairs - energy use, power production, and efficiency were all evaluated in stair climbing at different speeds

- Ball launches from ramps - different types of balls were launched from Hot Wheels ${ }^{\mathrm{TM}}$ ramps, with launch parameters used to estimate landing parameters - inaccuracies had to be explained

- Newton's Cradle - students evaluated what was happening in a Newton's Cradle, and then came up with their own interesting movement pattern and analyzed it

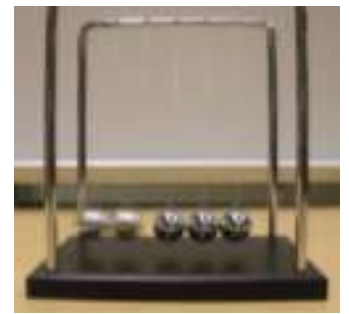

Figure 3 - Newton's Cradle: two balls swinging

- Shooting pool - similar to the Newton's Cradle exercise, students went to the local pub and analyzed various ways of hitting billiard balls for a practical study in impact dynamics 
- Meccano ${ }^{\mathrm{TM}}$ machines - students created simple machines out of Meccano ${ }^{\mathrm{TM}}$ that converted one type of motion (e.g. linear) to another (e.g. rotational) - students then developed a textbooklike question about their own machine, and solved it (they did this with both a kinematic and a kinetic question) e.g. http://youtu.be/luEFZPwEfOs
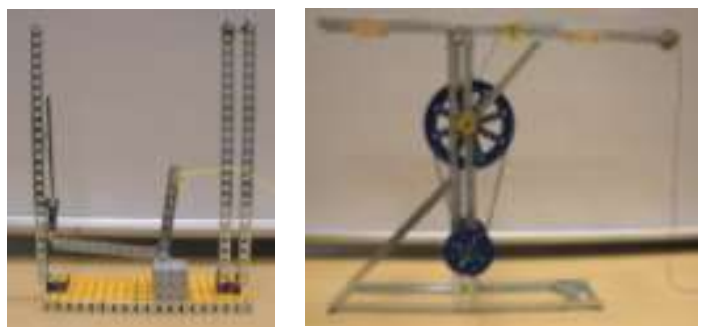

Figure 4 - Meccano ${ }^{\mathrm{TM}}$ Machines

\subsection{Projects}

Towards the end of the term, students completed term projects. The broad topic choices were i) develop a daily dynamic demo (with appropriate background material), ii) develop a new lab exercise (with appropriate example solutions) or iii) solve a set of challenging problems with a topical theme. Approximately two thirds of the students chose a DDD or lab exercise project and most of those were focused on a sport. Specifically, some of these projects dealt with:

- Lead climbing - a pair of students showed video of a lead climber falling off the wall, imparting an impulse to the belayer and a pendular motion towards the wall

- Hockey - three students illustrated lab exercises on ice, including relative motion (passes), impulses (shots) and projectile (puck) motion constrained by the locations of an obstacle and a target

- Pulleys - a pair of students built a frame out of Mecanno and attached a couple of pulleys - they illustrated various lab exercises that could be performed on the apparatus including physical validation of rope equations, evidence and measurement of static friction in the system and pros/cons of various rope/pulley configurations

- Basketball - three students illustrated lab exercises with a basketball and hoop, including projectile motion at a target, impacts/impulse/momentum, and projectile motion over an obstacle

- Slingshots - a pair of students illustrated lab exercises using a slingshot apparatus that explored the issues of spring linearity, impulse, KE/PE and projectile motion

\subsection{Quizzes, Assignments, Midterm, and Final}

Each of the three quizzes over the term examined student knowledge of important facts, terms and concepts. A couple of sports-related questions appeared on each quiz. Similarly, several of the questions on assignments (at least in the first 2/3rds of the course) involved sports. On the midterm, one question focused on a soccer pass over an opponent and onto the foot of a teammate. On the final exam, one question involved a basic hockey pass while another looked at a rail cam at a speed skating venue. A bonus question asked students to indicate where and how a runner should place their feet while running downhill on an icy trail, and then to justify their answer.

\section{DISCUSSION}

Overall, the sport theme to the course was well received. Students generally liked the tie-in to things they kinesthetically understood and cared about. It was also seen as a practical application that they could relate well to in a "real life" context. Several groups worked sports into projects, without any prodding or encouragement. The theme made some concepts easier to visualize for some students, as sports was seen as a natural fit with dynamics. On the other hand, at least one student did not like sports and therefore did not like the theme while another felt it was a waste of time because it would never be used in industry. A couple of others were ambivalent, or didn't notice it much, and suggested different themes such as "mechanical devices". Many noted the decline in sport examples in the last few weeks of the term.

The daily dynamic demos (DDDs) were generally quite popular. The majority of students seemed to appreciate them, with the exception of one student who preferred to see the situation described on the board in equation form and one other that resented the fact that no marks were associated with them (apparently they overlooked related questions on various tests). Several students commented positively on the visual nature of their learning and how it was a "clear" way of understanding theory. A few students noted how the demos were easily recalled versus textbook material. Students noted how the demos helped them understand the value of the material in the real world. One noted that they were a nice alternative to traditional learning. A few students did note that some DDDs weren't that effective at facilitating learning.

Virtually every one of the DDDs was a favourite of at least a couple of students. The most popular ones seemed to be those that were accessible but not trivial. Even the rolling ball was quite popular, possibly because the students had to try and describe its motion (in groups). The most popular were the "instructor on a pulley rope", 
the mogul skiing, curling, rolling wheel with LED, and spinning Meccano ${ }^{\mathrm{TM}}$.

One student noted that they looked forward to the DDD each class, as it was a new instructional element that they'd never really been exposed to before. They noted that before the course they had never been called upon to describe what was happening in real life using academic concepts. The DDDs helped them develop that skill, and there was some evidence of it on the final exam with the bonus question which most students got, of those who attempted it. One of the objectives of introducing the DDDs was to develop their physical intuition for dynamics. This effort appeared to be fairly successful. However, one student complained that only in a few cases was this intuition translated into equations and mathematical formulations. For this reason, they felt the DDDs were not as helpful as they could have been.

The labs elicited unique positive and negative comments. Admittedly, this was a heavy course. The lab work overloaded some students and there was resentment of the workload associated with lab reports. However, the actual lab exercises were mostly regarded favourably. Students commented positively on group problem solving aspects, real world relevance, sports tie-ins, independence of the problem solving process, and the application and critique of learned concepts. A small group suggested labs took up too much time for the learning that occurred.

When asked if these elements (theme, DDDs and lab exercises) assisted their learning, all but one student said "yes". However, precisely what motivated them to say yes did vary. One said that only the DDDs helped. Another said that sports helped because they played sports. Yet another student appreciated links to real life.

Suggested general course improvements for next year included an example DDD on wheel slippage, shorter (or no) labs, and more problem solving on the board.

\section{CONCLUSIONS AND FUTURE WORK}

The sports theme worked well for many, but the key may not have been "sports", per se. It may have been simply a theme that students could relate to and use as a foundational basis for understanding what they see in real life. Clearly, sports worked well, but not for all. And it was difficult to apply this theme later in the term, when other themes (such as mechanical devices) might have been more appropriate and also quite effective.

In terms of the DDDs, a pruning process will be undertaken. Top DDDs will be retained while those favoured by only one person will be replaced with new ones. Furthermore, more DDDs will be followed up with mathematical treatments to close the loop on their problem solving skills, as not all students seemed to be able to translate their new skill of describing motion in dynamics terms into a mathematical description.
Regarding the labs, not many of the lab exercises were explicitly sport focused and that seemed to work fine. Indeed, the running exercises were the least popular. To manage course workload, lab write-ups may need to be scaled down, however.

One of the most persistent suggestions during the term and at the end was that more problems should have been solved as examples on the board. While not directly relevant to the issues discussed in this paper, this idea does have one very important implication. The instructor has a finite amount of time with the students. More problem solving of the conventional textbook sort means less time for other activities like DDDs. Yet most of the students appreciated the DDDs. Perhaps the theory discussions could then be cut? But theory is important and it could be argued that it would irresponsible to not review it. Could this component of instruction be taped and uploaded online to free up class time or could problem solving be handled this way? It would appear that the "real life" problem solving inherent in the DDDs and lab exercises may be a new course element for which no time is available. Without the ability to offload some of these instructional elements to the web, this is probably true. But we have that capability now, and this author intends to expand his use of it.

As a final point relevant to further analysis of class use of these teaching methods, a more methodologically rigorous approach to some of the issues revealed in this qualitative overview could be useful in sharpening the accuracy of the conclusions. The use of triangulation as used in other forms of qualitative research, for example, could help to validate explanations for what seems to be occurring $[2,3]$.

\section{Acknowledgements}

The author would like to thank the students of ENGR 3349 for their participation in the course and for their feedback on its components, and Dr. Janice Miller Young for her editorial assistance and general help with the course.

\section{References}

[1] Meriam, J.L. and Kraige, L.G. Engineering Mechanics: Dynamics, $6^{\text {th }} \mathrm{Ed}-$ SI Version, John Wiley \& Sons, 2007

[2] Duffy, Mary E., Methodological Triangulation: A Vehicle for Merging Quantitative and Qualitative Research Methods, J Nursing Scholar, Vol. 19, \#3, pp 130-133, 1987

[3] Breitmayer, B.J., Ayres, L. and Knafl, K.A., Triangulation in Qualitative Research: Evaluation of Completeness and Confirmation Purposes, J Nursing Scholar, Vol. 25, \#3, pp 237-243, 1993 